\title{
Assessing the Role of Human Recombinant Hyaluronidase in Gravity-Driven Subcutaneous Hydration: The INFUSE-LR Study
}

\author{
JAY R. THOMAS, M.D., Ph.D., ${ }^{1}$ RICHARD C. YOCUM, M.D., ${ }^{2}$ MICHAEL F. HALLER, Ph.D., ${ }^{2}$ \\ and CHARLES F. von GUNTEN, M.D., Ph.D. ${ }^{1}$
}

\begin{abstract}
Background: Subcutaneous hydration has potential advantages over intravenous. Despite studies supporting the efficacy and safety of subcutaneous hydration it has not been studied extensively to date either with or without hyaluronidase.

Objectives: To compare flow rate, tolerability, and safety of gravity-driven subcutaneous fluid administration with and without recombinant human hyaluronidase (rHuPH20) in healthy volunteers.

Design: Randomized, double-blind, placebo-controlled, within-subject trial.

Setting: Contract research organization.

Participants: Fifty-four volunteers.

Intervention: 24-gauge angiocatheters were placed subcutaneously in both upper arms. Each arm received rHuPH20 (150 U, $750 \mathrm{U}$, or $1500 \mathrm{U})$ or equal volume saline placebo. Immediately, $400 \mathrm{~mL}$ Lactated Ringer's (LR) solution was gravity-infused from a $100 \mathrm{~cm}$ height. In the pilot stage, 5 subjects also received a similar intravenous infusion.

Measurements: Primary outcome was time to infuse $400 \mathrm{~mL}$ LR. Secondary outcomes included discomfort assessments, edema, arm circumference, time to recover to baseline arm circumference, subject and investigator global preference, and adverse events.

Results: rHuPH20 $150 \mathrm{U}, 750 \mathrm{U}$, and $1500 \mathrm{U}$ yielded mean flow rates of $383 \pm 119 \mathrm{~mL} / \mathrm{hr}, 518 \pm$ $154 \mathrm{~mL} / \mathrm{hr}$, and $494 \pm 136 \mathrm{~mL} / \mathrm{hr}$, respectively, compared to their respective placebo rates of $82 \pm$ $30 \mathrm{~mL} / \mathrm{hr}, 148 \pm 57 \mathrm{~mL} / \mathrm{hr}$, and $124 \pm 50 \mathrm{~mL} / \mathrm{hr}$. rHuPH20 was well tolerated.

Conclusions: In volunteers, clinically relevant fluid volumes can be rapidly delivered subcutaneously with $\mathrm{rHuPH} 20$ in a well-tolerated manner without a pump. These findings suggest that this method of hydration could potentially replace intravenous infusions in many clinical settings; further studies with rHuPH20, in patients, are warranted.
\end{abstract}

\section{INTRODUCTION}

$\mathbf{P}$ ARENTERAL HYDRATION is usually delivered intravenously. Yearly, practitioners in U.S. hospitals place more than 25 million intravenous (IV) catheters. ${ }^{1}$ Placement requires skill, yet patients often endure failed at- tempts due to difficult venous access (DVA) associated with skin pigmentation, vein sclerosis, fragility, collapse, or obesity or vasovagal reaction due to stress. IV catheter complications include malfunction, thrombosis, infection, and extravasation, which decrease systemic access and increase the cost of care. ${ }^{2-4}$

\footnotetext{
${ }^{1}$ San Diego Hospice \& Palliative Care, San Diego, California.

${ }^{2}$ Halozyme Therapeutics, Inc., San Diego, California.
} 
Subcutaneous (SC) hydration may avoid many IV complications and costs. ${ }^{5}$ Results from two studies indicated that the cost of cannulae is reduced by approximately two thirds with SC versus IV hydration. ${ }^{6,7}$ Because SC catheter placement requires less skill, patients and caregivers can develop expertise. SC catheter sites minimize bleeding and thrombosis risk. If infection occurs, it is typically localized. ${ }^{8}$ Studies support their continuous use for $5-12$ days. ${ }^{9,10}$ Moreover, neither heparin nor intermittent or continuous saline is needed to maintain catheter patency. There are many placement sites: upper chest, arms, upper back, abdomen, and thighs. While SC hydration can potentially decrease complications associated with IV catheter placement, it does not eliminate the role for IV catheters. In the past, SC hydration was linked to hypovolemic shock, but this complication was likely due to electrolyte-free solutions. ${ }^{11,12}$ A systematic review found SC administration of electrolyte-containing fluids to be safe. ${ }^{12}$

The SC space consists of collagen surrounded by "ground substance" including hyaluronan, a glycosaminoglycan. Hyaluronan's gel-like consistency is a major barrier to SC diffusion. Hyaluronidase hydrolyzes hyaluronan, temporarily reducing this barrier, for 24-48 hours. ${ }^{13}$ Early literature suggested hyaluronidase could enhance gravity-driven SC hydration by increasing flow rates. ${ }^{14}$ The Food and Drug Administration (FDA) determined that hyaluronidase is "effective for enhancing the dispersion and absorption of other injected drugs" and "for hypodermoclysis" (SC fluid administration). ${ }^{15}$ However, the role of hyaluronidase in SC hydration remains unclear.

Lipschitz et al. ${ }^{16}$ demonstrated that a subcutaneously delivered isotonic crystalloid solution was quickly and completely systemically absorbed. Constans et al. ${ }^{17}$ infused a $500 \mathrm{~mL}$ solution (5\% glucose, $2 \mathrm{~g} \mathrm{NaCl}$, and $0.375 \mathrm{~g} \mathrm{KCl}$ ) SC over 2 hours into patients' bilateral thighs preceded by hyaluronidase 100 $\mathrm{U}$ or placebo. Patients qualitatively reported no difference in thigh pain, but the hyaluronidase thigh's circumference was significantly smaller. Bruera et al. ${ }^{18}$ administered a $500 \mathrm{~mL}$ solution (two-thirds $5 \%$ glucose and one-third normal saline) subcutaneously over 1 hour preceded by hyaluronidase $150 \mathrm{U}$ or placebo. At this rate, there were no significant pain or edema differences. The authors concluded that hyaluronidase was unnecessary for routine pump-driven bolus SC hydration.

These studies used purified bovine hyaluronidase, which shares only partial human homology and has caused allergic reactions and rarely anaphylaxis. ${ }^{19} \mathrm{Al}-$ lergenicity concerns have limited its use. Human hyaluronidase, $\mathrm{PH} 20$, resides on a sperm's surface where it facilitates fertilization by depolymerizing oocyte hyaluronan. Recombinant human hyaluronidase, rHuPH20, is soluble and active under physiologic conditions. Intradermally injected rHuPH20 ( $n=100$ human volunteers) showed no allergenicity compared to placebo. ${ }^{20}$

Currently, SC hydration is limited by uncertainty about fluid delivery volumes and rates, questions regarding the need for hyaluronidase, and concerns about patient discomfort. This study assessed the flow rate, tolerability, and safety of gravity-driven SC fluid administration $\pm \mathrm{rHuPH} 20$.

\section{METHODS}

\section{Subject enrollment}

Independent Investigational Review Board, Inc. approved the protocol. Paid volunteers provided written informed consent and enrolled during November through December 2005 at a contract research organization (West Coast Clinical Trials, Long Beach, CA).

Inclusion criteria consisted of age 18 to 60 (inclusive), normal vital signs, venous access in 1 forearm, decision-making capacity, no fluid intake for 12 hours prior to study infusions, normal metabolic panel $\left(\mathrm{Na}^{+}\right.$, $\mathrm{K}^{+}, \mathrm{Cl}$, bicarbonate, blood urea nitrogen [BUN], creatinine, glucose, $\mathrm{Ca}^{2+}$, aspartate transaminase [AST], alanine transaminase [ALT], alkaline phosphatase, total bilirubin, albumin, and total protein), and, if female, a negative pregnancy test. Exclusion criteria included edema, upper extremity pathology that could interfere with study outcomes, cardiovascular disease, rales, coagulopathy, and allergy to hyaluronidase, bee, or vespid venom.

\section{Protocol}

The study had two stages. Stage 1 generated pilot data $(n=5)$ by comparing SC infusion to IV. Pilot study SC flow rate (mean \pm standard deviation $[\mathrm{SD}]$ ) with and without hyaluronidase was used to confirm the estimated stage 2 sample size. Stage 2 was powered to definitively assess SC flow rate \pm hyaluronidase. Stage 2, cohort 1 used hyaluronidase $150 \mathrm{U}$. If toxicity was acceptable, accrual for cohort 2 (1500 U) would be initiated. Accrual for cohort 3 (750 U) was initiated if cohort 2 mean flow rate with hyaluronidase was $20 \%$ or more than that of cohort 1 with acceptable toxicity.

Subjects received 24-gauge angiocatheters (BD Insyte Autoguard, [BD, Franklin Lakes, NJ] 0.75 inch) 


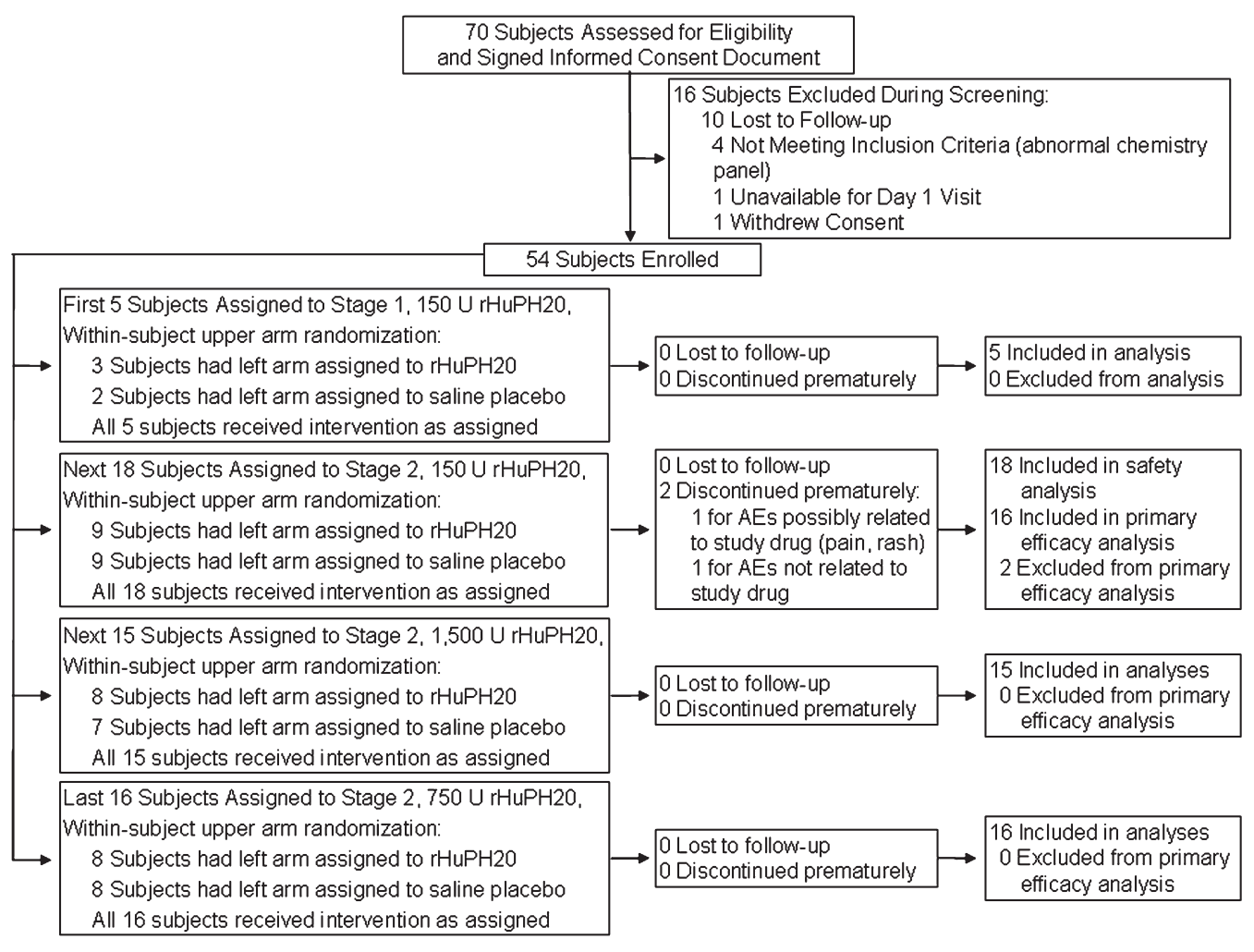

FIG. 1. Flow of subjects in the study.

subcutaneously in symmetrical positions on both proximal upper extremities at a 30-degree angle in a cephalad direction at the midline of the lateral aspect of the arm. The catheter was advanced to its hub, the metal stylus was removed, leaving the silastic tubing in place, and secured.

A 500-mL Lactated Ringer's (LR) bag (Na 130 $\mathrm{mEq} / \mathrm{L}, \mathrm{Cl} 109 \mathrm{mEq} / \mathrm{L}, \mathrm{K} 4 \mathrm{mEq} / \mathrm{L}$, Ca $3 \mathrm{mEq} / \mathrm{L}$, Lactate $28 \mathrm{mEq} / \mathrm{L}$; Baxter International Inc., Deerfield, IL) was hung from a digital scale attached to an infusion pole. The infusate's meniscus was placed $100 \mathrm{~cm}$ above its respective catheter. Infusate-primed tubing (V14234 IV Administration Set, 15 drops per milliliter, B-Series Set, B. Braun Medical Inc., Bethlehem, $\mathrm{PA})$ connected the bag to the catheter. In vitro from a $100-\mathrm{cm}$ height, the gravity-driven flow rate through the resistance of the tubing and 24-gauge catheter was approximately $1 \mathrm{~L} / \mathrm{hr}$ (Halozyme Therapeutics, San Diego, CA; data on file).

Prior to the study, staff not involved in study conduct, randomly allocated each subject's arms to the interventions by a manual method. A pharmacist prepared two identical syringes of equal volume rHuPH20 (hylenex $150 \mathrm{U} / \mathrm{mL}$; Baxter International Inc., Deerfield, IL) or normal saline placebo. Infusate bags with tubing were weighed at baseline. Immediately before each infusion, the randomized, double-blind dose of equal volume rHuPH20 (150 U, $750 \mathrm{U}$, or $1500 \mathrm{U})$ or its matched placebo $(1 \mathrm{~mL}, 5 \mathrm{~mL}$, or $10 \mathrm{~mL})$, respectively, were delivered simultaneously by steady push over 1 minute via a Y-port proximal to each arm's catheter site. Immediately, gravity-driven LR 400-mL infusions were begun. The primary outcome variable, LR flow rate, was monitored by noting the weight of the infusate bag at protocol-specified time points (flow rate $[\mathrm{mL} / \mathrm{hr}])=(($ base weight - final weight in $\mathrm{gm}) *$ $(1 \mathrm{~mL} / 1 \mathrm{gm}))$ / (finish time - start time [hours]). In stage 1 , intravenous flow rate was monitored identically.

Secondary outcomes and upper arm photographs were obtained at protocol-specified time points. The investigator rated edema, rash, and catheter site leakage on a $0-4$ point scale, and obtained arm circumference at the catheter site, halfway between the catheter and the olecranon process, and halfway between the catheter and the inferoposterior aspect of the acromion at baseline, mid-infusion each arm (once 200 $\mathrm{mL}$ had infused), at end of infusion each arm, and then every 30 minutes until the circumference in each arm returned to within $5 \%$ of the baseline circumference. 


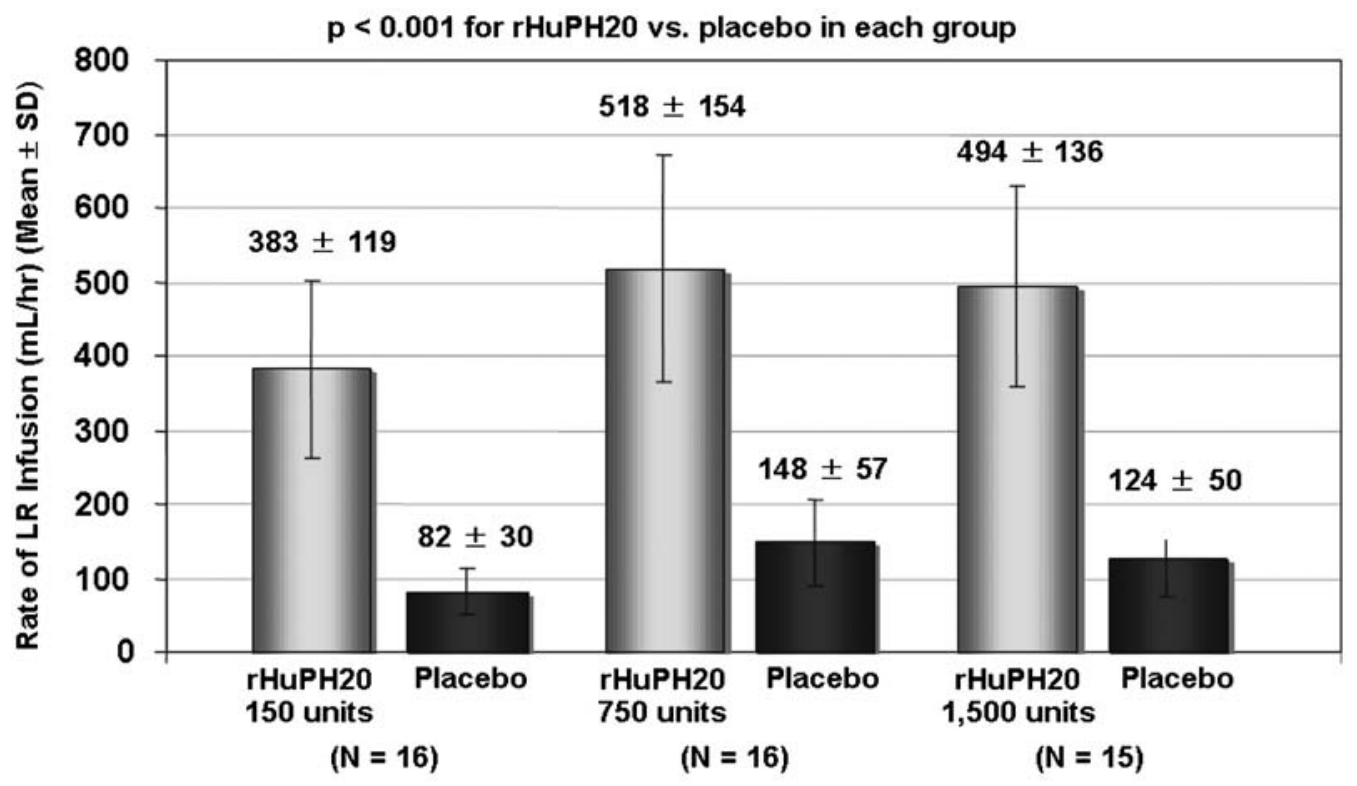

FIG. 2. Mean flow rates for subcutaneously administered infusion of Lactated Ringer's.

The time when the circumference returned to within $5 \%$ of baseline was recorded. Subjects rated discomfort on a $100-\mathrm{mm}$ visual analog scale (VAS) $(0=$ no discomfort; $100=$ worst discomfort). Subjects and the investigator separately ranked their global preferences for the infusions. All adverse events (AEs) were captured from the time of initial exposure through 28 days. Subjects underwent post-infusion evaluations at 1 and 28 days.

\section{Statistical analyses}

Assuming flow rate doubling and a SD of 42 minutes or less, a cohort sample size of 15 would permit detection of a significant difference using an unpaired $t$-test with an $\alpha$ of 0.05 and a $\beta$ of 0.20 . Statistical analyses were performed using SAS (SAS Institute, Cary, NC) to evaluate McNemar's test for binomial variables, the sign rank test for global preferences, and paired t-tests for all other variables. Flow rate was determined on an as-treated basis; safety analysis was performed on an intent-to-treat basis. Continuous data were presented as mean $\pm \mathrm{SD}$. While the normality assumption was not rejected for any cohort, additional nonparametric paired analyses were also performed. Both the signed rank test and sign test confirmed results of the paired $t$-test.

\section{Role of funder}

The sponsor was involved in study design and data collection and management, statistical analysis, data interpretation, and manuscript preparation. Drs. Thomas and von Gunten retained final content control.

\section{RESULTS}

\section{Subjects}

Subject flow through the trial is presented in Figure 1 . Five and 49 volunteers were enrolled in stage 1 and stage 2, respectively. Overall, $52 \%$ were female. Mean age was $28.9 \pm 7.8$ years. Mean body mass index was $28.1 \pm 6.1 \mathrm{~kg} / \mathrm{m}^{2}$. Subjects were evenly distributed among the stages and cohorts of the study.

Stage 1: Pilot. In the 5 stage 1 subjects, mean flow rates with and without $\mathrm{rHuPH} 20$ were $313 \pm 151$ $\mathrm{mL} / \mathrm{hr}$ and $108 \pm 59 \mathrm{~mL} / \mathrm{hr}$, respectively $(p=0.049)$. The pilot means and SDs confirmed the stage 2 sample size estimate. The mean IV flow rate in these 5 subjects was $809 \pm 295 \mathrm{~mL} / \mathrm{hr}$. Two IVs infiltrated and required restarting during the infusion. Four of the 5 stage 1 subjects stated a global preference for SC infusion over IV infusion. Subcutaneous infusion with rHuPH20 was their first choice or tied for first in 4 subjects.

Stage 2: Flow Rate. In every subject tested, rHuPH20 injection preceding gravity-driven LR infusion-enabled flow rate approximately $250 \%$ to $400 \%$ com- 


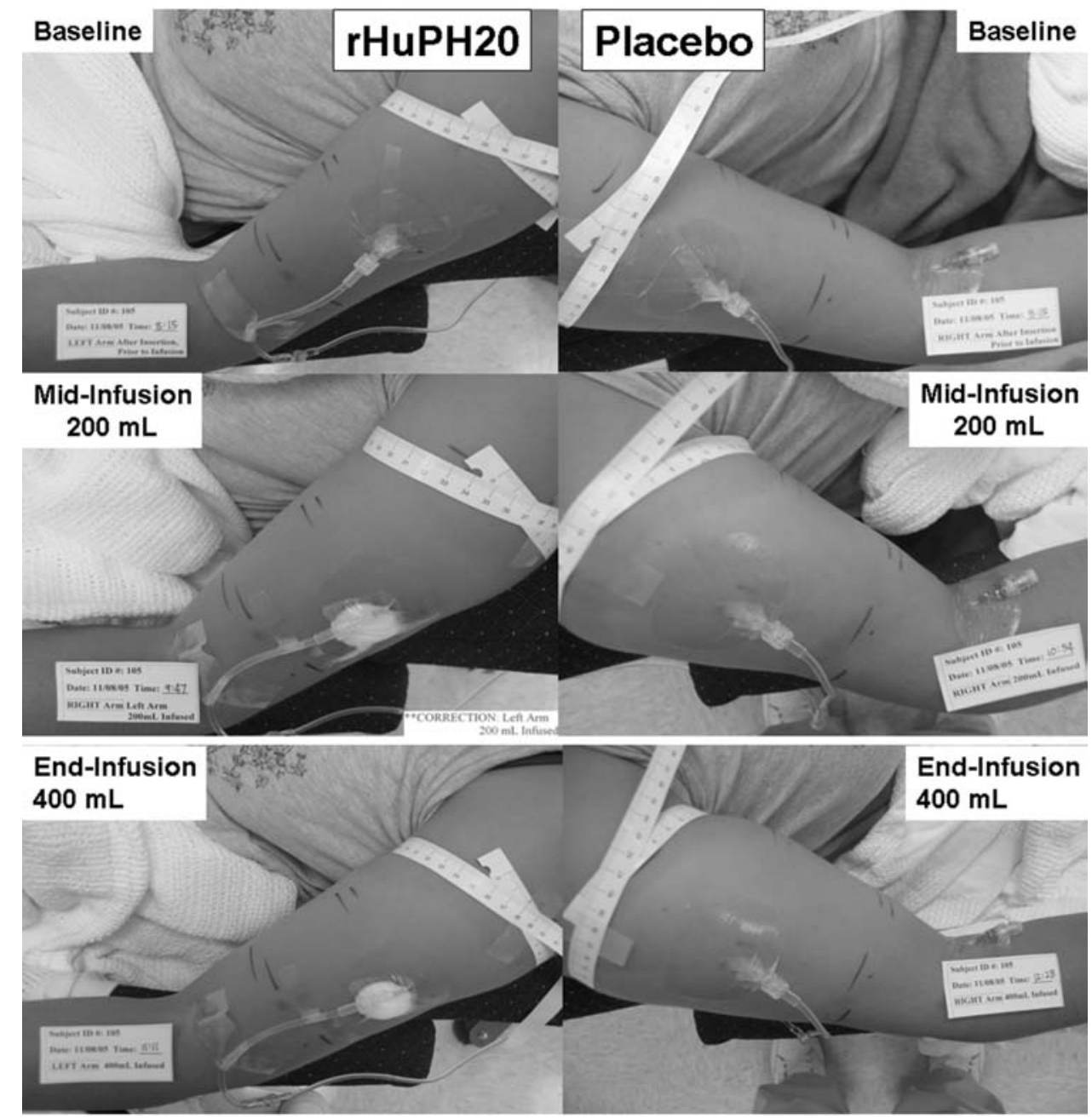

FIG. 3. Edema in a representative subject's arm during infusion.

pared to an infusion without rHuPH20. Mean flow rates for $\mathrm{rHuPH} 20$ versus placebo (unpaired analyses) are shown in Figure 2. The mean paired differences in flow rate between placebo and rHuPH20 (rHuPH20 placebo) were $301 \mathrm{~mL} / \mathrm{hr} \pm 117$ (150 U), 369 $\mathrm{mL} / \mathrm{hr} \pm 157$ (750 U), and $371 \mathrm{~mL} / \mathrm{hr} \pm 148$ (1500 U). As noted in Figure 1, 2 subjects dropped out. Exclusion of these 2 subjects from the efficacy analysis did not influence the results.

Tolerability. The investigator graded edema on a 0-4 scale, meaning no, minimal, mild, moderate, or severe edema, respectively. Figure 3 shows a representative subject's edema. At all rHuPH20 doses and all time points during infusions, hyaluronidase arms yielded significantly less edema. Figure 4 shows the proportion of subjects with moderate and severe edema according to placebo or rHuPH20 and by dosing cohort. Despite the difference in investigator rated edema, arm circumference measurements around the site of infusion did not appear to show clinically meaningful increases in either treatment group, and on average represented less than a $10 \%$ increase from baseline. Arm circumference measurements were also used as an indication of systemic fluid absorption. Each rHuPH20 dose led to a statistically significant decrease in time to baseline circumference (Fig. 5).

Volunteers subjectively rated their discomfort on a 100-mm VAS. Overall, subjects rated only minimal pain in both arms. Without $\mathrm{rHuPH} 20$, the pain score was $9.6 \pm 15.3 \mathrm{~mm}$; with rHuPH20, the score was significantly reduced to $5.8 \pm 10.7 \mathrm{~mm}(p<0.002)$. Finally, the subjects and investigator rated their global preference for the SC infusion with or without rHuPH20. For the $150 \mathrm{U}, 750 \mathrm{U}$, and 1,500 U dose cohorts, respectively, the subjects' preference for the infusion with rHuPH20 was $88 \%(p=0.002), 100 \%$ $(p<0.001)$, and $87 \%(p=0.007)$, and the investiga- 


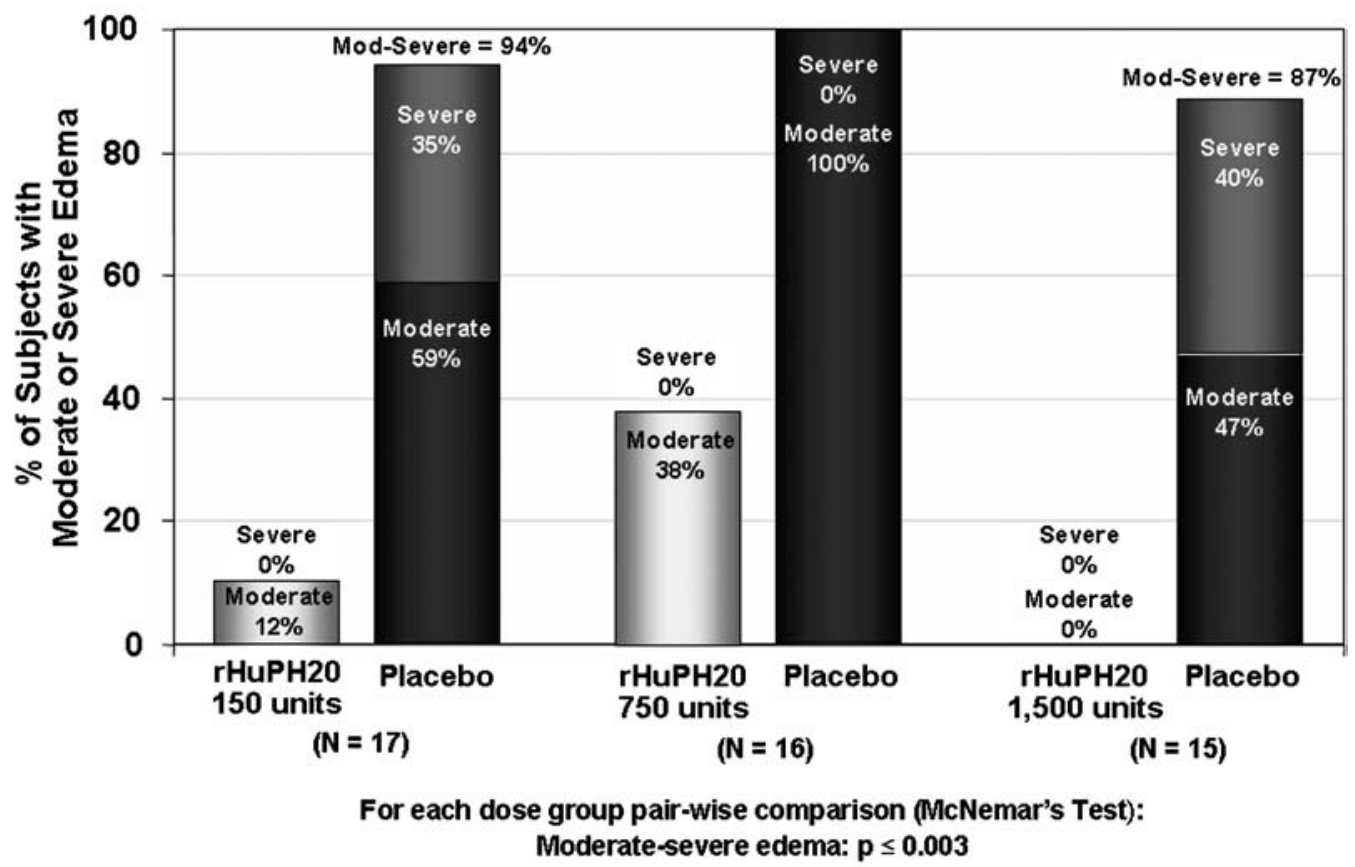

FIG. 4. Incidence of moderate and severe edema.

tor's preference was 94\% $(p<0.001), 100 \%(p<$ $0.001)$, and $80 \%(p=0.035)$.

\section{Safety}

Overall, treatment-emergent AEs occurring through 28 days after treatment were mild to moderate in severity, localized to the infusion sites, and evenly distributed between placebo and rHuPH20 (Table 1). There were no severe or serious AEs. One subject developed a papular rash around the rHuPH20 infusion site and on the torso. It was asymptomatic and resolved spontaneously in approximately 10 minutes.

\section{CONCLUSION/DISCUSSION}

This randomized, double-blind study showed that rHuPH20 significantly enhanced gravity-driven subcutaneous infusion flow rates versus placebo. A clin-

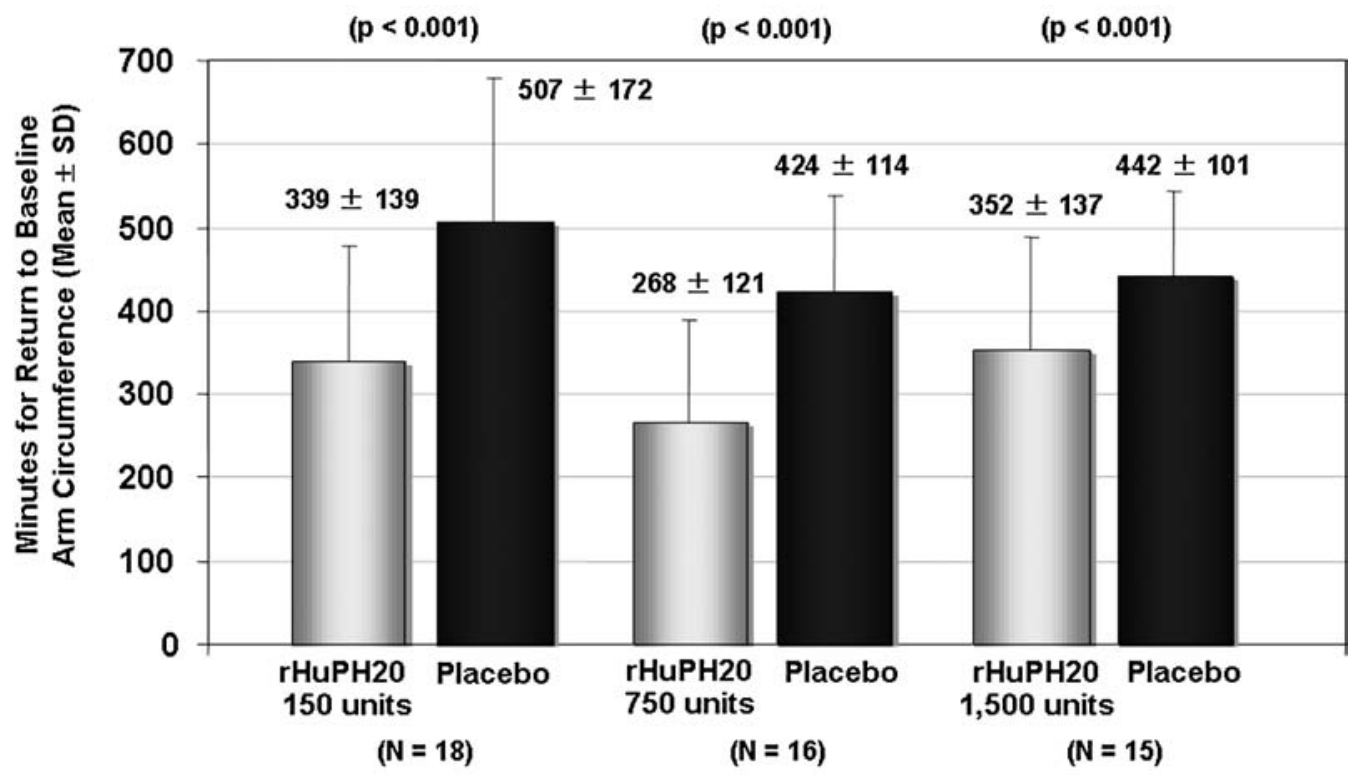

FIG. 5. Time to recovery to baseline arm circumference. 
Table 1. Incidence of Adverse Events: All Adverse Events Regardless of Relatedness to Study Drug Experienced by $\geq 2$ Subjects Each in Either Treatment Group in Stage 2

\begin{tabular}{|c|c|c|c|c|c|c|c|c|}
\hline \multirow[b]{2}{*}{$\begin{array}{l}\text { MedDRA SOC/ } \\
\text { Preferred Term }\end{array}$} & \multicolumn{2}{|c|}{ Cohort $1(\mathrm{n}=18)$} & \multicolumn{2}{|c|}{ Cohort $3(\mathrm{n}=16)$} & \multicolumn{2}{|c|}{ Cohort $2(\mathrm{n}=15)$} & \multicolumn{2}{|c|}{$\begin{array}{c}\text { All cohorts } \\
(\mathrm{n}=49)\end{array}$} \\
\hline & $\begin{array}{l}\text { rHuPH2O } \\
150 \mathrm{U}\end{array}$ & Placebo & $\begin{array}{l}\text { rHuPH2O } \\
750 \mathrm{U}\end{array}$ & Placebo & $\begin{array}{l}\text { rHuPH2O } \\
1500 \mathrm{U}\end{array}$ & Placebo & rHuPH2O & Placebo \\
\hline $\begin{array}{l}\text { General disorders and administrative } \\
\text { site conditions }\end{array}$ & $38.9 \%$ & $50.0 \%$ & $81.3 \%$ & $87.5 \%$ & $73.3 \%$ & $73.3 \%$ & $63.3 \%$ & $69.4 \%$ \\
\hline Infusion/injection/catheter site pain & $27.8 \%$ & $38.9 \%$ & $75.0 \%$ & $75.0 \%$ & $46.7 \%$ & $60.0 \%$ & $49.0 \%$ & $57.1 \%$ \\
\hline Infusion/injection site burning & $11.1 \%$ & $5.6 \%$ & $6.3 \%$ & $12.6 \%$ & $13.3 \%$ & $6.7 \%$ & $10.2 \%$ & $8.2 \%$ \\
\hline Injection site paraesthesia & $0.0 \%$ & $0.0 \%$ & $18.8 \%$ & $12.5 \%$ & $0.0 \%$ & $6.7 \%$ & $6.1 \%$ & $6.1 \%$ \\
\hline Injection site anaesthesia & $11.1 \%$ & $5.6 \%$ & $0.0 \%$ & $6.3 \%$ & $0.0 \%$ & $0.0 \%$ & $4.1 \%$ & $4.1 \%$ \\
\hline Infusion/catheter site erythema & $5.6 \%$ & $0.0 \%$ & $0.0 \%$ & $0.0 \%$ & $6.7 \%$ & $13.3 \%$ & $4.1 \%$ & $4.1 \%$ \\
\hline Infusion site induration & $0.0 \%$ & $11.1 \%$ & $0.0 \%$ & $0.0 \%$ & $0.0 \%$ & $0.0 \%$ & $0.0 \%$ & $4.1 \%$ \\
\hline
\end{tabular}

All adverse events were captured from the time of initial exposure to study drug through the 28-day follow-up assessment.

ically relevant volume of an isotonic solution was delivered in a reasonable time frame in a well-tolerated manner. This clinical trial was the first to assess the role of rHuPH20 in SC fluid administration. As opposed to studies done with animal hyaluronidase, the fluid was not pump-driven in a set time. The flow rate was dependent on the fluid height above the catheter, tubing resistance, catheter gauge, and tissue resistance. A 24-gauge catheter was chosen for its minimal discomfort and its ability to support flow rates up to 1 $\mathrm{L} / \mathrm{hr}$ under ex vivo experimental conditions. The observed flow rates \pm hyaluronidase did not reach this limit in subjects, implying tissue resistance is rate-limiting for flow. Larger gauge catheters have not been studied under these same parameters, and therefore there are no data to address whether or not larger gauge catheters might allow significantly increased flow, with or without hyaluronidase. The upper extremity was used because of patient preference and accessibility, which facilitated examination and measurement, and given its limited expandibility, was a good test of edema and discomfort with bolus fluid delivery. Whether other body sites would yield similar results deserves further study. LR solution was chosen as an isotonic solution that could help replenish $\mathrm{Na}^{+}$and $\mathrm{K}^{+}$, as well as volume, in dehydrated patients.

Gravity-driven mean flow rates with rHuPH20 ranged from 383 to $518 \mathrm{~mL} / \mathrm{hr}$ over the three dose cohorts compared to 82 to $148 \mathrm{~mL} / \mathrm{hr}$ for placebo. rHuPH20 dosing over an order of magnitude (150 U, $750 \mathrm{U}$, and $1500 \mathrm{U}$ ) showed no linear dose response. Placebo flow rate increased as the sequential dosing cohorts were performed temporally. The reason for this increase is unknown, but hypothesized to be attributable to 2 factors. First, improving flow rates for both $\mathrm{rHuPH} 20$ and placebo may be related to improving skill in placing and maintaining the SC cath- eters as the study progressed. Second, the volume injected prior to the infusions ( $1 \mathrm{~mL}, 5 \mathrm{~mL}$, or $10 \mathrm{~mL}$ ) differed among the dosing cohorts. Injecting different volumes over 1 minute created different sized subcutaneous "pockets," which may have affected flow rates. Nevertheless, within each subject, the 250 to $400 \%$ increase in flow rate between an individual's arms was seen in all cohorts. It is likely that the volume and rate of hyaluronidase-enabled gravity-driven SC infusion can be increased by raising the height of the infusate, increasing the catheter size, or using a different infusion site.

In this study, isotonic solution was gravity-delivered subcutaneously with $\mathrm{rHuPH} 20$ at a mean rate approaching $500 \mathrm{~mL} / \mathrm{hr}$. This volume and rate are similar to what was pump-delivered in Bruera's study with or without hyaluronidase of a one third normal saline solution given subcutaneously in the chest or abdomen. In contrast to Bruera's study, the infusions generated a significant edema difference. Without rHuPH20, tissue contours were grossly distorted and indurated; with $\mathrm{rHuPH} 20$, tissue contours were preserved and induration was minimal. Perhaps the studies' different solution tonicities explain the contrast in study findings. Interestingly, despite the gross edema differences, clinically meaningful increases from baseline in arm circumference were not observed. Presumably, rHuPH20-mediated hydrolysis of hyaluronan led to even fluid distribution, with increase in circumference to a small extent, but minimizing local edema. Consistent with previous literature, this volume of SC infusion produced minimal discomfort \pm rHuPH20. Although hyaluronidase significantly decreased discomfort according to the VAS, the absolute difference between rHuPH20 and placebo for this measurement was probably not clinically relevant. ${ }^{21}$ Both subjects and the investigator preferred the $\mathrm{rHuPH} 20$ 
enabled infusion. The study was not designed to probe the reason for this preference.

The fact that the mean flow rates and local edema were observably different between the arm that received $\mathrm{rHuPH} 20$ and the arm that received placebo raises the possibility that the study became functionally unblinded. However, flow rate is an objective measure not easily subject to bias. This study might also be criticized for its "as-treated" analysis as opposed to an "intent-to-treat" analysis. However, it is justifiable to include subjects who actually are treated and complete measurements. ${ }^{22-24}$ Only 2 subjects were excluded from the primary analysis. In both cases it was because the subject had premature discontinuation of both LR infusions and therefore could not be evaluated because it was not reasonably possible to extrapolate the flow rate for a $400 \mathrm{~mL}$ infusion. In this within-subject controlled study where each subject served as their own control, the premature discontinuation rate remained balanced between the 2 interventions. This study was carried out in healthy volunteers, which may hinder generalization of findings to frail elderly patients.

The rHuPH20-enabled infusions were well tolerated, although the study was not powered to detect infrequent events. However, rHuPH20 is FDA-approved and animal hyaluronidases have been overall safe in millions of administrations. There have been allergic reactions and rarely anaphylaxis with animal hyaluronidases. The human enzyme, $\mathrm{rHuPH} 20$, is up to 100 -fold purer than animal hyaluronidases based on activity and is expected to have a lower rate of allergic reaction in single and repeat dosing; however, more study is needed. Adverse events, which were either mild or moderate in severity, were approximately equally distributed between the $\mathrm{rHuPH} 20$ and placebo arms and there was no rHuPH20 dose relationship for AEs. There were no severe or serious AEs and no allergic reactions that required intervention.

In summary, an $\mathrm{rHuPH} 20$-enabled, gravity-driven SC infusion delivered a clinically relevant volume of an isotonic solution in a clinically relevant time frame in a well-tolerated manner. Clinically, larger fluid volumes ( 1 to 2 liters) need to be delivered in a timely manner. Thus, there is a need to further define the largest rHuPH20-enabled fluid bolus that can be gravity-delivered subcutaneously in a relevant time frame and what body areas can tolerably accommodate that volume. The extent to which hyaluronidase can enhance continuous gravity-driven SC infusions, such as maintenance hydration at rates of approximately 125 $\mathrm{mL} / \mathrm{hr}$ or more, must also be assessed. Animal studies have shown that dermal hyaluronan is reconstituted within 24 hours of hyaluronidase administration. ${ }^{25}$ If infusions continue 24 hours or more, subsequent hyaluronidase dosing and timing to maintain effect need to be defined. Because hyaluronidase increases subcutaneous absorptive surface area, it will be interesting to assess the rate and extent that coadministered medications are absorbed. If SC absorption can approach that of IV, the delivery of medications, including opioids and antibiotics, could be enhanced. Finally, there is a need for a cost effectiveness analysis of SC versus IV hydration. Nevertheless, this study supports the possibility of using gravity-driven rHuPH20-enabled SC infusions as replacements for pump-driven IV fluid therapy.

\section{ACKNOWLEDGMENTS}

Dr. Kerry Barker performed the statistical analysis as an employee of Baxter International Inc. Dr. Gregory Frost provided input into the design of the study and Linda Heiner facilitated the study's conduct.

Dr. Thomas and Dr. von Gunten have no personal financial disclosures. They and their institutions have received funding for research studies with human hyaluronidase and to teach health care professionals about the subcutaneous route of drug delivery and may receive additional funding of this nature in the future. Dr. Thomas was the principal investigator of these trials. Dr. Yocum and Dr. Haller are employees of Halozyme Therapeutics, Inc., which funded the study. Baxter International Inc. supported the study with study documents, the database, and statistical analysis.

Clinical Trial Registration: 〈www.ClinicalTrials. gov identifier: NCT0031151

\section{REFERENCES}

1. Soifer NE, Borzak S, Edlin BR, Weinstein RA: Prevention of peripheral venous catheter complications with an intravenous therapy team: Randomized controlled trial. Arch Intern Med 1998;158:473-477.

2. Kurul S, Saip P, Aydin T: Totally implantable venous-access ports: Local problems and extravasation injury. Lancet Oncol 2002;3:684-692.

3. Monreal M, Davant E: Thrombotic complications of central venous catheters in cancer patients. Acta Haematol 2001;106:69-72.

4. Sasson M, Shvartzman P: Hypodermoclysis: An alternative infusion technique. Am Fam Physician 2001;64:15751578.

5. Dasgupta M, Binns MA, Rochon PA: Subcutaneous fluid infusion in a long-term care setting. J Am Geriatr Soc 2000;48:795-799. 
6. Challiner YC, Jarrett D, Hayward MJ, al Jubouri MA, Julious SA: A comparison of intravenous and subcutaneous hydration in elderly acute stroke patients. Postgrad Med J 1994;70:195-197.

7. O'Keeffe ST, Lavan JN: Subcutaneous fluids in elderly hospital patients with cognitive impairment. Gerontology 1996;42:36-39.

8. Yap LK, Tan SH, Koo WH: Hypodermoclysis or subcutaneous infusion revisited. Singapore Med J 2001;42:526529.

9. Fainsinger RL, MacEachern T, Miller MJ, Bruera E, Spachynski K, Kuehn N, Hanson J: The use of hypodermoclysis for rehydration in terminally ill cancer patients. J Pain Symptom Manage 1994;9:298-302.

10. Macmillan K, Bruera E, Kuehn N, Selmser P, Macmillan A: A prospective comparison study between a butterfly needle and a Teflon cannula for subcutaneous narcotic administration. J Pain Symptom Manage 1994;9:82-84.

11. Frisoli JA, de Paula AP, Feldman D, Nasri F: Subcutaneous hydration by hypodermoclysis. A practical and low cost treatment for elderly patients. Drugs Aging 2000;16:313319.

12. Rochon PA, Gill SS, Litner J, Fischbach M, Goodison AJ, Gordon M: A systematic review of the evidence for hypodermoclysis to treat dehydration in older people. J Gerontol A Biol Sci Med Sci 1997;52:M169-M176.

13. Hylenex Prescribing Information. Deerfield, IL: Baxter Healthcare Corporation, 2006.

14. Gaisford W, Evans DG: Hyaluronidase in paediatric therapy. Lancet 1949;2:505-507.

15. Food and Drug Administration. Federal Register 1970;35: 14800-14801.

16. Lipschitz S, Campbell AJ, Roberts MS, Wanwimolruk S, McQueen EG, McQueen M, Firth LA: Subcutaneous fluid administration in elderly subjects: Validation of an underused technique. J Am Geriatr Soc 1991;39:6-9.

17. Constans T, Dutertre JP, Froge E: Hypodermoclysis in dehydrated elderly patients: local effects with and without hyaluronidase. J Palliat Care 1991;7:10-12.

18. Bruera E, Neumann CM, Pituskin E, Calder K, Hanson J: A randomized controlled trial of local injections of hyaluronidase versus placebo in cancer patients receiving subcutaneous hydration. Ann Oncol 1999;10:1255-1258.
19. Ebo DG, Goossens S, Opsomer F, Bridts CH, Stevens WJ: Flow-assisted diagnosis of anaphylaxis to hyaluronidase. Allergy 2005;60:1333-1334.

20. Yocum RC, Kennard D, Heiner LS: Assessment and implication of the allergic sensitivity to a single dose of recombinant human hyaluronidase injection: A double-blind, placebo-controlled clinical trial. J Infus Nurs 2007;30:293299.

21. Kelly AM: The minimum clinically significant difference in visual analogue scale pain score does not differ with severity of pain. Emerg Med J 2001;18:205-207.

22. Peng B, Hayes M, Resta D, Racine-Poon A, Druker BJ, Talpaz M, Sawyers CL, Rosamilia M, Ford J, Lloyd P, Capdeville R: Pharmacokinetics and pharmacodynamics of imatinib in a phase I trial with chronic myeloid leukemia patients. J Clin Oncol 2004;22:935-942.

23. Swanson J, Gupta S, Lam A, Shoulson I, Lerner M, Modi N, Lindemulder E, Wigal S: Development of a new oncea-day formulation of methylphenidate for the treatment of attention-deficit/hyperactivity disorder: Proof-of-concept and proof-of-product studies. Arch Gen Psychiatry 2003;60:204-211.

24. Wiltshire H, Hirankarn S, Farrell C, Paya C, Pescovitz MD, Humar A, Dominguez E, Washburn K, Blumberg E, Alexander B, Freeman R, Heaton N: Pharmacokinetic profile of ganciclovir after its oral administration and from its prodrug, valganciclovir, in solid organ transplant recipients. Clin Pharmacokinet 2005;44:495-507.

25. Bookbinder LH, Hofer A, Haller MF, Zepeda ML, Keller GA, Lim JE, Edgington TS, Shepard HM, Patton JS, Frost GI: A recombinant human enzyme for enhanced interstitial transport of therapeutics. J Control Release 2006;114:230-241.

Address reprint requests to: Jay Thomas, M.D. San Diego Hospice \& Palliative Care $43113 r d$ Avenue San Diego, CA 92103

E-mail: jthomas@sdhospice.org 